\title{
Sheltering or Evacuation? Role of Emergency Monitoring in Nuclear Disaster Control
}

\author{
Nagoya University, Hiromi Yamazawa \\ Aomori Prefectural Nuclear Power Safety Center, Hideki Kimura \\ Fukushima Prefecture Government, Yoshihiro Koyama \\ Tohoku University, Toshiki Iwasaki
}

\begin{abstract}
This report is a compilation of the planning session of the Health Physics and Environmental Science Subcommittee of the Atomic Energy Society of Japan that took place in the 2015 fall meeting. In addition to a lecture focused on the details and issues of monitoring required by the Nuclear Emergency Response Guidelines, lectures were conducted on the status of monitoring performed by the local prefecture at the time of the Fukushima Nuclear Power Plant Accident under the constraints of an enormous natural disaster, on lessons learned therefrom, and on the current status of reviews. In addition, the Meteorological Society of Japan, which specializes in atmospheric dispersion, conducted a lecture on the usefulness and limitations of predictive calculations and explained proposals made by the society after the accident. There are advantages and disadvantages to both the monitoring and atmospheric dispersion predictions. Therefore, overall, the session discussed that the complementary use of both will lead to a more effective disaster prevention scheme.
\end{abstract}

KEYWORDS: nuclear accident, environmental monitoring, emergency operation, emergency response scheme, atmospheric dispersion prediction

\section{Introduction}

The Nuclear Regulation Authority established the "Nuclear Emergency Response Guidelines" (hereinafter, referred to as the "guidelines") in 2012 to ensure smooth and reliable disaster responses by utility companies, government and local administrative organizations, and relevant public agencies in the event of a nuclear emergency; the current guidelines were set through the fifth process of revisions made in August 2015. The purpose of a disaster response is to prevent deterministic effects and minimize probabilistic effects of radiation. The enactment of and revisions to these guidelines were implemented based on a reflection upon numerous existing problems with regard to nuclear power disaster responses before the Fukushima Daiichi Nuclear Power Plant Accident e.g., lack of a resident-based viewpoint,

DOI : 10.15669/fukushimainsights.Vol.1.312

(C) 2021 Atomic Energy Society of Japan. All rights reserved.

Originally published in Journal of the Atomic Energy Society of Japan (ISSN 1882-2606), Vol. 58, No. 2, p. 115-120 (2016)

in Japanese. (Japanese version accepted: November 16, 2015) 
inadequate preparation of systems and materials, and the non-clarity of the decision-making process. As a basic idea, "countermeasures from a resident-based viewpoint," "continuous provision of information," and a "review for optimization" were clearly expressed in the preamble of the guidelines.

In these guidelines, the basic policy of determining the disaster response was established on the basis of the measured air dose rate, and mechanisms were established to enable decision making with regard to response measures based on clear judgment criteria. From the perspective of the ease of understanding, the guidelines are thought to be reasonable. However, it is important to clarify whether the purpose of the countermeasures based on these guidelines can be sufficiently achieved. Excluding the question of whether the judgment criteria are appropriate, the practicality of the scheme depicted by the guidelines heavily depends on how successful the emergency environmental monitoring is. The reasonability of the judgment criteria must be discussed separately. It would be worth pointing out here that determining plume countermeasures or restrictions of food and drinking water solely on the basis of air dose rates is difficult.

In the session, the primary topics of discussion included information that must be obtained through emergency monitoring to understand the environmental situation; this information serves as the basis for decision making, feasibility, and problems of monitoring. The session was configured to also include discussions on the capability of relevant atmospheric dispersion predictions. First, Hideki Kimura, Director of the Aomori Prefectural Nuclear Power Center, who participated in discussions related to monitoring when enacting the guidelines, discussed the key points and issues associated with the guidelines from the perspective of implementing monitoring. Next, Yoshihiro Koyama, Head of the Fukushima Prefectural Nuclear Power Safety Response Section at the time of the Fukushima nuclear power accident, who was in charge of controlling the response for the overall prefecture, discussed the current status and reflections. Furthermore, Toshiki Iwasaki, President of the Meteorological Society of Japan, conducted lectures on (a) recognition among research fields specializing in meteorology and atmospheric dispersion with respect to the capability and usefulness of atmospheric dispersion predictions in the event of a nuclear power accident and (b) proposals made thus far by the Meteorological Society regarding nuclear power disaster prevention.

Numerous discussions from the floor arose with regard to each of the lectures, and it is thought that the purpose of sharing the current status and problems of emergency monitoring and atmospheric dispersion predictions with all participating members was sufficiently achieved. I served as the chairman of the discussions, but due to my lack of skill in guiding discussions, summarization discussions of the overall session were not fully achieved for the most part. However, from the perspective of a chairman, it is evident that there exist respective advantages and disadvantages related to both monitoring and atmospheric dispersion predictions, and thus, an overall scheme utilizing both monitoring and atmospheric dispersion predictions in a complementary manner must be considered. I also pointed out my apprehensions about the weaknesses of the current scheme, which adopts only monitoring, as a rough conclusion of the overall session. In particular, it is unlikely that a nuclear emergency would occur alone, and therefore, we must seriously consider whether the current scheme is truly feasible and effective from the perspective of hardware and manning key personnel in a case of a nuclear power plant accident in conjunction with a major natural disaster, similar to the Fukushima nuclear power plant accident.

(Hiromi Yamazawa) 


\section{Monitoring Required by the Guidelines}

\section{Current Status of Environmental Radiation Monitoring}

Environmental radiation monitoring in Japan can be broadly classified into surveys of the impact of radiation on areas surrounding a nuclear power facility and surveys of environmental radiation levels throughout Japan. With regard to the former, the surveys are primarily conducted by the local government, where the nuclear power facility is located, along with the cooperation of the utility company. With regard to the latter, the surveys are conducted by 47 prefectures in Japan as projects commissioned by the Government of Japan. An advanced and specialized radioactivity analysis is conducted by the Japan Chemical Analysis Center. In both cases, the survey details include continuous measurements of air dose rates by monitoring posts and analysis and measurements of radioactive materials in environmental samples, e.g., atmospheric dust, drinking water, soil, seawater, and food products.

\section{Emergency Monitoring as per the Guidelines}

The idea of environmental radiation monitoring in the event of an emergency (hereinafter referred to as "emergency monitoring") was presented in the guidelines that were enacted in October 2012 and in supplementary reference documents based on the accident at Tokyo Electric Power Company's (TEPCO) Fukushima Daiichi Nuclear Power Plant that occurred in association with the Great East Japan Earthquake in March 2011.

In the initial response stage of an emergency situation, the guidelines classify emergency situations into three categories according to the status of the nuclear power facility: an alert, a site area emergency, and a general emergency. In addition, as zones in which response measures are devised with priority in the event of a nuclear emergency, in the case of a nuclear power plant, a precautionary action zone (PAZ) with a radius approximately $5 \mathrm{~km}$ from the nuclear power plant is established as a zone for the preparation of precautionary protection measures according to the emergency activity level (EAL) from the stage before the emission of radioactive materials into the environment. An urgent protective action-planning zone (UPZ) with a radius of generally $30 \mathrm{~km}$ from the power plant is established for the preparation of emergency protection measures based on the EAL and the operational intervention level (OIL).

In an alert, the Government of Japan, local governments, nuclear power utility companies, and relevant designated public institutions implement preparations for emergency monitoring. Emergency monitoring during site area emergency is primarily implemented by the Emergency Monitoring Center (EMC). If radioactive materials are emitted into the environment from the nuclear power facility, necessary measures are implemented based on the emergency monitoring results. As the judgment criteria thereof, the OIL is designated for measurable values, e.g., the air dose rates and the concentration of radioactive materials in environmental samples.

The purposes of emergency monitoring are as follows:

(1) Collect information related to the status of environmental radiation caused by a nuclear emergency.

(2) Provide materials that can be used to implement protection measures determined on the basis of the OIL.

(3) Provide materials for the evaluation of the impact of radiation on the environment and inhabitants caused by the nuclear accident. 
Among these, in the initial monitoring, priority is given to the materials required for the determination of protection measures.

Regarding monitoring for OIL1 (air dose rate of $500 \mu \mathrm{Sv} / \mathrm{h}$ ), as first priority, air dose rates continuously measured by fixed monitoring stations and portable monitoring posts are primarily used. As necessary, monitoring is implemented using monitoring cars and survey meters. The methods and locations of monitoring for an OIL2 (air dose rate of $20 \mu \mathrm{Sv} / \mathrm{h}$ ) are the same, but airborne monitoring is also conducted to supplement the measurements at the fixed observation stations.

Regarding monitoring for OIL6, which is a criterion for radioactive materials in foods and drinks, first, regions wherein the concentration of radioactive materials in foods and drinks must be measured (regions exceeding an air dose rate of $0.5 \mu \mathrm{Sv} / \mathrm{h}$ ) are identified through air dose rate measurements by the fixed observation stations and aerial monitoring. As for OIL6, initial settings of concentration are prescribed with regard to radioactive iodine, radioactive cesium, plutonium, alpha-ray emitting transuranium nuclides, and uranium targeting "drinking water, milk, and dairy products" and "vegetables, grains, meat, egg, fish, etc.," and analyses and measurements are performed thereon.

Regarding protection measures outside the UPZ, for which consideration was given to the impact of the radioactive plume emitted from the nuclear power facility, sheltering instructions are expanded to a certain range beyond the UPZ based on the conditions of the facility and the emergency monitoring results. When it has been confirmed that the radioactive plume has passed the said range, the instructions for sheltering are to be quickly lifted. To this end, information within the site and at the site boundary must be obtained, an overview of the path of the radioactive plume must be understood through monitoring using monitoring equipment within the UPZ, and mobile monitoring techniques, e.g., carborne monitoring and airborn monitoring, must be used to gather information that will contribute toward determining the passage of the radioactive plume in the expanded protection area.

\section{Future Issues and Outlook}

To correspond to the aforementioned monitoring process required by the guidelines, the system has been reinforced for measuring air dose rates and a system for publicizing the monitoring information has been developed. However, to further improve the effectiveness of monitoring, we must address the following issues.

With respect to measuring air dose rates, to cope with natural disasters, it is necessary to multiplex communication lines, increase the soundness of monitoring facilities, and establish a system for promptly enabling the replacement of devices and alternative measurements with spare devices. In addition, to gain an overall understanding of the radiation levels and estimate the path of the plume, efforts are needed to build an analysis methodology that combines measurements and numerical simulations.

To handle numerous environmental samples in the measurement of radioactive nuclides, it is recommended to develop a measurement system that transects the administrative field and establish pre-processing and measurement manuals that reflect the experiences gained through this accident. In addition, to evaluate the internal dose received by the inhabitants and understand the impact from the facility, a system for sampling and measuring radioactive nuclides in the atmosphere and a system for analyzing alpha-ray emitting nuclides (plutonium, etc.) and beta-ray emitting nuclides (strontium 90, etc.) must be reinforced.

Finally, going forward, continuing efforts are essential to "acquire data" through an effective and efficient monitoring system, to "utilize data" through the visualization of evaluation 
results and measures to notify inhabitants, and particularly to "raise personnel" by establishing a comprehensive monitoring education and support system for enhancing and reinforcing monitoring.

(Hideki Kimura)

\section{Initial Emergency Monitoring Response by Fukushima Prefecture in the Fukushima Daiichi Nuclear Power Plant Accident}

\section{Preface}

In the Fukushima Prefecture (hereinafter referred to as the "prefecture"), efforts were made to reinforce the preparation for natural disasters with respect to environmental radiation monitoring (hereinafter referred to as "emergency monitoring") in the event of a nuclear emergency. However, when the accident occurred at the Tokyo Electric Power Company's (TEPCO) Fukushima Daiichi Nuclear Power Plant (hereinafter referred to as "Daiichi Power Plant") on March 11, 2011 (hereinafter, the month and year will be omitted unless otherwise noted) due to the Great East Japan Earthquake, responses had to be implemented under major constraints, such as securing equipment, materials, and key personnel in general. In this section, I will present an overview of the status of the initial emergency monitoring activities in the prefecture.

\section{Status When the Earthquake Disaster Occurred}

\section{(1) Status of Damage to the Facility Due to the Earthquake and Tsunami}

The Fukushima Prefectural Nuclear Power Center (hereinafter referred to as "prefectural center") is located adjacent to the Fukushima Prefecture Nuclear Emergency Response Center (hereinafter referred to as "off-site center") within the town of Okuma located approximately $4.9 \mathrm{~km}$ to the west-southwest of the Daiichi Power Plant, and when the nuclear emergency occurred, the prefectural center was supposed to serve as an emergency monitoring center. Immediately after the earthquake, which exceeded a seismic intensity of 6 , the entire region suffered a power outage and a water outage due to the breakage of water pipes and public telephone lines. In addition, cell phone service became difficult to use for the most part and the function of these and other public infrastructures was lost. While significant damage to the building of the prefectural center itself was not observed and emergency power generators operated without any problems, printers, FAX machines, and other devices in the office and the measurement laboratory toppled over and additional damage was observed, such as dislocation of the shields of two of four germanium semiconductor detectors, resulting in an inability to perform measurements. Furthermore, with regard to the "environmental radiation telemetry systems" that performed focused monitoring at a total of 23 environmental radiation measurement stations installed in six towns around the nuclear power plant, four of the measurement stations were carried away by the tsunami. In addition, at around 4:42 pm on March 11, the designated wired communication line was interrupted. With the exception of one measurement station located on the site of the prefectural center, data could not be collected at the remaining measurement stations.

While in the midst of efforts to understand the damage status of the facility at the 
prefectural center (eight full-time personnel) and to restore the center, notification of the occurrence of an emergency situation based on the Act on Special Measures (Act on Special Measures Concerning Response to Environmental Contamination by Radioactive Material Released from the Accident of the Nuclear Power Plant Caused by the Tohoku District-off the Pacific Ocean Earthquake) was received and the team began advancing with emergency monitoring preparations.

Note that at that time, with regard to external communication with the prefectural center, email could be intermittently used until the early morning of March 12 but could not be used from that point onward. Even the designated communication lines prepared for use in the event of a disaster gradually became more difficult to use, and from the afternoon of March 12, satellite cell phones became the only means of communication for the most part.

\section{(2) Status of Gathering Key Personnel for Emergency Monitoring}

In the nuclear power disaster prevention plan of the prefecture, personnel from the prefectural center correspond the preparation and intensive monitoring as an intial respnse, then, an emergency monitoring team was to be organized from the personnel (total of 52 people) dispatched from local prefectural administrative organizations, local towns, and TEPCO, after which support was to be received from government agencies on a national level to enhance the system (total of 120 people). However, immediately after the earthquake, it became difficult to dispatch key personnel from the prefecture, the towns, and local organizations owing to the earthquake and tsunami response. From the early morning of March 12, i.e., the following day, key personnel dispatched from TEPCO (5 people), the Japan Atomic Energy Agency ( 7 people), and the prefecture ( 20 people) were gradually assembled and emergency monitoring activities, e.g., measuring outdoor air dose rates, were initiated. Furthermore, on March 13, personnel dispatched from the Ministry of Education, Culture, Sports, Science and Technology (MEXT) also participated. By March 14, emergency monitoring activities were being continuously implemented by approximately 30 personnel.

However, with the expansion of the evacuation range, the range subject to surveys also exceeded a range of $10 \mathrm{~km}$ from the power plant, which was the priority range for implementing emergency monitoring. Wireless communications between the prefectural center and the monitoring cars became difficult, and it was unclear how replacement personnel could be secured and how the vehicles could be refueled. These and several other issues gradually became obstacles to continuing the monitoring activities. Therefore, on the afternoon of March 14 , following discussions with the offsite center radiation team, the continuation of activities at the local site was discontinued. On the evening of the same day, the prefectural center was evacuated and the base for emergency monitoring activities was moved to the Fukushima city.

\section{Measurement Results Obtained via Emergency Monitoring}

\section{(1) Emergency Monitoring Results of the Prefectural Center}

On the early morning of March 12, the instructed evaluation range that had been within $3 \mathrm{~km}$ of the Daiichi Power Plant based on instructions issued at 9:23 pm the previous day was expanded to a $10 \mathrm{~km}$ range at 5:44 am and three survey teams for measuring air doses while traveling in monitoring cars were assembled for understanding the situation of the entire area within $10 \mathrm{~km}$ of the Daiichi Power Plant. Surveys were then conducted along National Highway No. 6 by primarily traveling in the north and south along the Pacific coast. The first survey on March 12 was conducted from 8 am to 9 am, and the second survey was conducted from 11:30 am to 1:30 pm. In each survey, air dose rates were measured at nine locations, 
atmospheric suspended dust was collected at three locations, and samples were brought back to the prefectural center, where they were analyzed for radionuclides. In the first survey, an increase in the air dose rate was observed to the north and northwest of the Daiichi Power Plant (the maximum value was $15 \mu \mathrm{Sv} / \mathrm{h}$ at a location $8 \mathrm{~km}$ to the northwest) and radioactive nuclides, e.g., iodine 131 (maximum of $166 \mathrm{~Bq} / \mathrm{m}^{3}$ of iodine 131), were confirmed from the samples of atmospheric suspended dust. In the vicinity of the main gate of the Fukushima Daiichi Nuclear Power Plant, the dose rate began to increase from around 4 am on March 12, but it was clear that the impact extended to a wide surrounding area.

At 5:39 pm on March 12, evacuation instructions were expanded to within a range of $10 \mathrm{~km}$ from the Fukushima Daini Nuclear Power Plant, and at 6:25 pm, the evacuation instructions were further expanded to within a $20 \mathrm{~km}$ radius. With a limited number of personnel and materials, deliberations regarding what type of emergency monitoring should be implemented were conducted with the off-site center radiation team. Then, on March 13, i.e., the next day, air dose rates were measured and atmospheric suspended dust samples were collected in a range from the city of Minamisoma to the city of Iwaki as well as from the Fukushima Daiichi Nuclear Power Plant to approximately $30 \mathrm{~km}$ to the north and south. The monitoring car of the Japan Atomic Energy Agency also traveled and conducted surveys from Minamisoma to Okuma and within $20 \mathrm{~km}$ in the northwest direction from the Daiichi Power Plant.

As a result, an increase in the air dose rate that exceeded $30 \mu \mathrm{Sv} / \mathrm{h}$ was confirmed in Minamisoma, the town of Namie, and the town of Futaba. Iodine 131 was also detected in the afternoon in the northern part of Iwaki.

These survey results were all reported to the radiation team of the offsite center, but the Nuclear and Industrial Safety Agency publicized only a part of them. As a result, it could not be said that the results were effectively utilized in formulating protection measures for the inhabitants, such as evacuation. In addition, in the prefecture, information sharing between the Nuclear Emergency Response Headquarters of the prefecture and the prefectural center was difficult and appropriate information was not provided to relevant organizations of the cities and towns in which evacuation was conducted. All measurement data obtained during March 11 to March 15 by the off-site center radiation team was disclosed on June 3.

Note that other than the four measurement stations that were washed away by the tsunami, four of the remaining 19 stations did not suffer power outages and the remaining 15 stations continued measurements until around March 15 when the fuel for the emergency power supply ran out. This fact became clear when data were collected later. These data were useful in analyzing the initial dispersion conditions of radioactive materials.

\section{(2) Monitoring Results Within the Prefecture}

To understand the radiation levels throughout the prefecture, independent of the surveys conducted by the prefectural center, when the nuclear emergency occurred, the prefecture decided to measure air dose rates using survey meters at local promotion bureaus established at seven locations within the prefecture. In addition, the results of the measurements initiated from March 11 to March 12 were summarized at the Nuclear Emergency Response Headquarters of the prefecture. The prefecture, with support from the monitoring organizations of other prefectures, deployed eight portable monitoring posts within a $20-50 \mathrm{~km}$ radius from the Fukushima Daiichi Nuclear Power Plant by March 13.

Through these surveys, air dose rate measurements were initiated immediately after $5 \mathrm{pm}$ on March 12 at the Prefectural Hamadori Local Promotion Bureau (Minamisoma) located approximately $25 \mathrm{~km}$ north-northwest of the Daiichi Power Plant and a dose rate increase of $20 \mu \mathrm{Sv} / \mathrm{h}$ was measured at $9 \mathrm{pm}$ on the same day. Dose rate increases were detected at the 
other six local promotion bureaus on the afternoon of March 15.

\section{Response Thereafter}

On and after March 16, from the government side, MEXT began coordinating the implementation of the environmental monitoring and publicizing the results. The prefecture set up a base for emergency monitoring at the Fukushima Branch (city of Fukushima) of the Prefectural Nuclear Power Center, and collaboration between the off-site center radiation team and the Nuclear Emergency Response Headquarters of the prefecture was finally secured. As a result, all efforts were devoted toward understanding the status of radioactive material contamination throughout the prefecture.

In addition, with regard to initial emergency monitoring activities, it was pointed out that, at prefectural headquarters, the handling of information from the System for Prediction of Environmental Emergency Dose Information (SPEEDI) and data from portable monitoring posts was inappropriate. Based on the lessons and experiences gained during the emergency phase of the earthquake, the prefecture is now endeavoring to revise the emergency monitoring system, with priority given to the following three points as initial response issues: "preparation of emergency monitoring resources and materials that can cope with natural disasters," "securing personnel for emergency monitoring in the event of a complex disaster," and "the public release of data when the off-site center is not functioning."

(Yoshihiro Koyama)

\section{Use of Numerical Prediction on Atmospheric Dispersion of Radioactive Materials Released from a Nuclear Power- Related Facility Accident (from a Working Group Report of the Meteorological Society of Japan)}

\section{Activities of the Meteorological Society of Japan}

After the accident at the Fukushima Daiichi Nuclear Power Plant (hereinafter referred to as the Fukushima Accident), the Meteorological Society of Japan established a "Working Group on Radioactive Material Dispersion in Association with an Accident at a Nuclear Power-Related Facility" (hereinafter referred to as the Working Group) to examine how to provide information when an accident involving the release of radioactive materials occurs. The Working Group concluded that a monitoring system must be established and dispersion predictions using numerical models must be used. On March 05, 2012, the Working Group announced the "Proposal on Countermeasures to Radioactive Material Dispersion in the Event of a Nuclear Power Facility Accident" under the name of the President of the Meteorological Society of Japan ${ }^{1)}$.

In the "Operation of SPEEDI" announced on October 08, 2014, the Nuclear Regulation Authority decided that "when determining protective measures, such as evacuations and temporary relocations in an emergency, calculation results through SPEEDI shall not be used" ${ }^{2}$. This differed from the opinion of the Working Group, and therefore, the Working Group assembled a "proposal related to reinforcing monitoring and prediction technology with regard to the atmospheric dispersion of radioactive materials in association with an accident at a 
nuclear power-related facility." As the Meteorological Society of Japan, the group proposed that a numerical model must be used ${ }^{3}$. At the same time, the Working Group announced a "plan for utilizing numerical prediction information related to the atmospheric dispersion of radioactive materials in association with an accident at a nuclear power-related facility" (hereinafter referred to as the "utilization plan") 4 .

From March 2015 to April 2015, the Nuclear Regulation Authority solicited public opinion regarding the guidelines (revision drafts) and the Meteorological Society of Japan submitted the opinion that numerical models should be used ${ }^{5)}$. The basic ideas of these proposals and the key points of the utilization plan assembled by the Meteorological Society of Japan and the Working Group thereof are presented below.

\section{Monitoring Data and Prediction Data}

Accurate ground level observations by monitoring posts are indispensable for monitoring actual conditions and should be proactively expanded. However, the following drawbacks should be acknowledged.

(1) The data represent currently existing conditions without lead time and their usefulness in planned evacuations are limited.

(2) The data are collected at ground level, and there is a possibility that contamination exists in regions wherein monitoring is not being implemented. Radioactive materials passing aloft might be overlooked.

(3) With accidents attributed to large-scale natural disasters, such as the Fukushima Accident, monitoring posts and communication networks will not necessarily function.

Atmospheric dispersion predictions of radioactive materials with a numerical model have a significant merit of being capable of issuing advance warnings (providing lead time). However, owing to estimation errors in source term and numerical calculation errors, the predicted absolute values have significant errors. Therefore, in the "utilization scheme," rather than absolute values, it is proposed to use the predicted values as relative indicators representing the spatiotemporal distributions of danger. The ability of numerical predictions to reproduce the approximate movement of radioactive materials in the air has been proven by the SPEEDI predictions ${ }^{6}$ that were implemented at that time as well as through the calculation model comparisons ${ }^{7)}$ made by the Science Council of Japan.

In a nuclear emergency, the monitoring data and numerical prediction data are in a mutually complementary relationship. Both should be maximally utilized to reduce damage.

\section{Key Points for Effectively Utilizing Numerical Predictions}

\section{(1) Forecasts Providing Lead Time and an Accurate Understanding of the Actual Conditions Are Both Necessary for Disaster Prevention}

Based on numerous experiences, disaster prevention systems for natural disasters have been established, in which situations are assessed by combining forecasts and actual conditions. In the case of typhoons, prediction information containing uncertainties, e.g., "tomorrow afternoon, strong typhoon winds will hit XX, and the winds could gain strength," and actual values, e.g., "today, at $3 \mathrm{pm}$, maximum wind speeds of $30 \mathrm{~m} / \mathrm{s}$ were recorded at XX," are both important disaster prevention details for reducing damage. Even in a nuclear emergency, all types of information should be used to build a scheme (disaster prevention system) for minimizing damage. 
(2) If Release Amounts are Unknown, Conduct Simulations with a Unit Amount of Release and Use the Results as Spaciotemporal Distribution of the Relative Risk

The assumption of a unit release corresponds to a worst case scenario, which may result in a false alarm, but will never fail to issue an alarm. This type of simulation also plays an important role in formulating emergency monitoring plans and in the evaluation of discrete monitoring data.

(3) The Suspension of Radioactive Materials in the Air near the Ground and the Wet Deposition are to be Separately Warned About; the Vertically Integrated Amount is used as the Potential Deposition Amount

In numerical predictions, the predicted amount of precipitation can be used to calculate the deposition amount, but prediction errors in precipitation amount distributions are large. As a result, they can cause deposition to be overlooked. Vertically integrated values for radioactive materials calculated without deposition are used as worst case scenarios (potential maximum deposition amounts) that do not overlook risk.

If a high concentration of radioactive materials is predicted in the air near the ground, issue a warning, e.g., "around the time of $\bigcirc \bigcirc$ near XX, there is a risk that the concentration of radioactive materials in the air near the ground could increase, and therefore, you must evacuate."

If a high potential deposition amount is predicted, issue a warning, e.g., "around the time of $\bigcirc \bigcirc$ near XX, there is a danger that the precipitation could Contain redioactive material." If rain has actually fallen, implement restrictions on water intake and circulation, and monitor the contamination status. If there is no precipitation, cancel the wet deposition warning.

(4) Considering the Uncertainty of Prediction Results, Assume Wider Potential Risk Areas in Terms of Time and Space

In the case of dispersion predictions for a nuclear emergency, it is important to assume worst case scenarios. It is important not to miss issuing warnings, even in the case of false alarms.

As described above, even if monitoring results are used in determining protection measures, e.g., evacuations and temporary relocations, numerical predictions can be used as forecasts for devising and evaluating monitoring plans. These predictions can also be used for avoiding unnecessary exposure during evacuation or other actions. In addition, as was learned from the Chernobyl Accident and the Fukushima Accident, even in regions relatively distant from the UPZ (radius: $30 \mathrm{~km}$ ), wet deposition can cause serious contamination. If the predicted value for the vertically integrated amount (potential deposition amount) and the values for actual precipitation conditions are used, regions with a high potential contamination can be significantly narrowed. It then becomes possible to efficiently specify the contaminated areas and implement countermeasures for food product screening in these areas. Numerical predictions provide various usage merits, and effective utilization of numerical predictions is anticipated.

(Toshiki Iwasaki)

\section{References}

1) Meteorological Society of Japan. Proposal Relating to Countermeasures to Radioactive Material Dispersion in the Event of a Nuclear Power Facility Accident. http://www.metsoc.jp/others/News/proposal_120305.pdf. 2012. [in Japanese]

2) Nuclear Regulation Authority. Operation of the System for Prediction of Environmental Emergency Dose Information (SPEEDI). http://www.nsr.go.jp/data/000027740.pdf. 2014. [in Japanese] 


\section{INSIGHTS CONCERNING THE FUKUSHIMA DAIICHI NUCLEAR ACCIDENT Vol. 1}

3) Meteorological Society of Japan. Proposal Relating to Reinforcing Monitoring and Prediction Technology with Regard to the Atmospheric Dispersion of Radioactive Material in Association with an Accident at a Nuclear Power-Related Facility. http://www.metsoc.jp/2014/12/17/2467. 2014. [in Japanese]

4) Meteorological Society of Japan Working Group. Plan for Utilizing Numeric Prediction Information Relating to the Atmospheric Dispersion of Radioactive Material in Association with an Accident at a Nuclear Power-Related Facility. http://www.metsoc.jp/default/wp-content/uploads/2014/12/teigen-201412.pdf. 2014. [in Japanese]

5) Meteorological Society of Japan, Board of Directors Meeting. Opinions Regarding Amendments to Nuclear Emergency Response Guidelines and Related Nuclear Regulation Authority Regulations. http://www.metsoc.jp/2015/03/31/3837. 2015. [in Japanese]

6) Chino M. Verification! prediction information provided by SPEEDI in Fukushima Nuclear Power Plant Accident - Information and timeliness, use in the future. J. Atomic Energy Society of Japan. 2013; 55:220-224. [in Japanese]

7) Science Council of Japan. Report. A Review of the Model Comparison of Transpotation and Deposition of Radioactive Materials Released to the Enviroment as a Result of the Tokyo Elecric Power Conpany's Fukushima Daiidhi Nuclear Power Plant Accident 103 pp. 